\title{
A CHARACTERIZATION OF BALLS THROUGH OPTIMAL CONCAVITY FOR POTENTIAL FUNCTIONS
}

\author{
PAOLO SALANI \\ (Communicated by Joachim Krieger)
}

\begin{abstract}
In this short note two unconventional overdetermined problems are considered. Let $p \in(1, n)$; first, the following is proved: if $\Omega$ is a bounded domain in $\mathbb{R}^{n}$ whose $p$-capacitary potential function $u$ has two homotetic convex level sets, then $\Omega$ is a ball. Then, as an application, we obtain the following: if $\Omega$ is a convex domain in $\mathbb{R}^{n}$ whose $p$-capacitary potential function $u$ is $(1-p) /(n-p)$-concave (i.e. $u^{(1-p) /(n-p)}$ is convex), then $\Omega$ is a ball.
\end{abstract}

\section{INTRODUCTION}

Let $n \geq 3, \Omega \subset \mathbf{R}^{n}$ and $p \in(1, n)$. The $p$-capacity of $\Omega$ can be defined as follows (see for instance [10, $§ 4.7$ ):

$$
\operatorname{Cap}_{p}(\Omega)=\inf \left\{\int_{\mathbf{R}^{n}}|\nabla v|^{p} d x: v \in C_{c}^{\infty}\left(\mathbf{R}^{n}\right), v(x) \geq 1 \forall x \in \Omega\right\},
$$

where $C_{c}^{\infty}\left(\mathbf{R}^{n}\right)$ denotes the set of functions from $C^{\infty}\left(\mathbf{R}^{n}\right)$ having compact support.

In the sequel $\Omega$ is a bounded open convex set. Then the above infimum is in fact a minimum which is realized by the (classical) solution $u$ of the following problem:

$$
\left\{\begin{array}{l}
\operatorname{div}\left(|\nabla u|^{p-2} \nabla u\right)=0 \quad \text { in } \quad \mathbf{R}^{n} \backslash \bar{\Omega}, \\
u(x)=1 \text { in } \bar{\Omega} \\
\lim _{|x| \rightarrow+\infty} u(x)=0 .
\end{array}\right.
$$

The function $u$ is called $p$-capacitary potential function of $\Omega$ and it holds

$$
\operatorname{Cap}_{p}(\Omega)=\int_{\mathbf{R}^{n} \backslash \bar{\Omega}}|\nabla u|^{p} d x .
$$

It is well known that if $\Omega$ is (bounded, open and) convex, then $u$ is quasi-concave, that is, all its superlevel sets

$$
\Omega(t)=\left\{x \in \mathbb{R}^{n}: u(x) \geq t\right\} \quad t \in(0,1]
$$

are convex; see $11,14,17$. In fact, if $\Omega$ is smooth and strictly convex, one could even expect $u$ to satisfy some stronger concavity property, in particular, $u$ could

Received by the editors October 28, 2012 and, in revised form, March 6, 2013.

2010 Mathematics Subject Classification. Primary 35N25, 35R25, 35R30, 35B06, 52A40.

Key words and phrases. Capacity, convex sets, power-concavity, Brunn-Minkowski inequality. 
be power concave. We recall here that a positive function $v$ is $\alpha$-concave, for some $\alpha \in[-\infty,+\infty)$, if

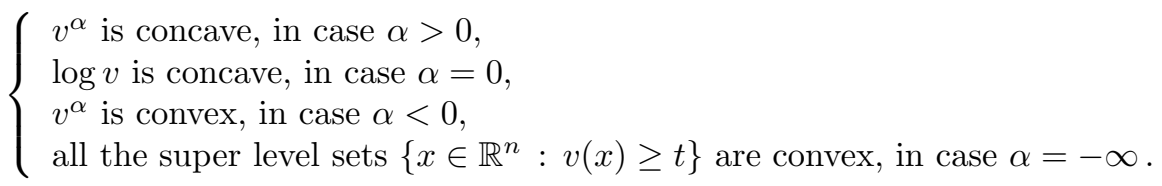

In the cases $\alpha=-\infty$ and $\alpha=0, v$ is also (and more usually) denoted quasi-concave and log-concave, respectively, while when $\alpha=1$ one is dealing with usual concave functions. if

Notice that, if $v \in C^{2}\left(\mathbb{R}^{n},[0, \infty)\right)$ and $\alpha>-\infty$, then $v$ is $\alpha$-concave if and only

$$
v \nabla^{2} v \leq(1-\alpha) \nabla v \otimes \nabla v \quad \text { in } \mathbb{R}^{n}
$$

in the sense of symmetric matrices.

From Jensen's inequality (or from (1.4) if $v$ is regular enough) it follows that if $v$ is $\alpha$-concave for some $\alpha>-\infty$, then $v$ is $\beta$-concave for every $\beta \leq \alpha$; and it is clearly quasi-concave too. Given a quasi-concave function $v$, it is then natural to ask whether it satisfies some better concavity properties and following [16] it makes sense to define the concavity number of $u$ as follows:

$$
\alpha(v)=\sup \{\beta \leq 1: v \text { is } \beta \text {-concave }\} .
$$

It is easily also seen that if $\alpha(v) \in \mathbb{R}$, then the above supremum is in fact a maximum. Please refer to 16 for more details on $\alpha$-concave functions.

Finally, since, as we said, when $\Omega$ is convex its $p$-capacitary potential $u$ is quasiconcave, we can set

$$
\alpha(\Omega, p)=\alpha(u)
$$

which we may call the p-capacitary concavity number of $\Omega$.

If $\Omega$ is sufficiently regular and strictly convex, one can expect that $\alpha(\Omega, p)>-\infty$ (see Section 5 for a partial discussion of this and related topics). Indeed, when $\Omega$ is a ball of radius $R>0$ centered at $x_{0}$ it is easy to find explicitly the solution of (1.2), that is,

where

$$
u(x)=R^{q}\left|x-x_{0}\right|^{-q}
$$

$$
q=\frac{n-p}{p-1},
$$

and it results to be $(-1 / q)$-concave.

In this short note we prove that nothing better is possible and that this power concavity is optimal among convex sets, in the sense that the property of $u^{-1 / q}$ to be convex characterizes balls. Precisely, the main result of this paper is the following.

Theorem 1.1. Let $p \in(1, n)$ and $\Omega$ be a bounded convex domain in $\mathbb{R}^{n}$. Then

$$
\alpha(\Omega, p) \leq-\frac{p-1}{n-p}
$$

and equality holds if and only if $\Omega$ is a ball.

To prove this theorem we will use three main ingredients:

- the first one is the Brunn-Minkowski inequality for $p$-capacity and its equality condition, proved in [3,4] for $p=2$ and in [8] for a generic $p$; 
- the second ingredient is an expression of $p$-capacity through the behavior at infinity of the potential function; see formula (2.4);

- the third ingredient is an easy relation existing between the $p$-capacity of a generic level set of $u$ and the capacity of $\Omega$; see formula (2.5).

In fact the second ingredient is needed to prove the following property, which has its own interest and is new, to our knowledge.

Theorem 1.2. If the solution $u$ of (1.2) has two homothetic convex level sets, then $\Omega$ is a ball.

In particular: if $u$ has a level set that is homothetic to $\Omega$ (and $\Omega$ is convex), then $\Omega$ is a ball. We recall here that two sets $A, B \subset \mathbb{R}^{n}$ are said homothetic if there exist $\rho>0$ and $\xi \in \mathbb{R}^{n}$ such that $B=\rho A+\xi$, i.e. if they are dilate and translate of each other.

Let us note here that, even though in this presentation Theorem 1.1 is somewhat more enlightening than Theorem 1.2. the former can in fact be seen just as a nice application of the latter.

To some extent, both the problems considered in Theorems 1.1 and 1.2 fall in the framework of overdetermined problems. An overdetermined problem usually consists in a Dirichlet problem given in an unknown domain, whose solution is required to satisfy some extra condition (classically a Neumann boundary condition) which is so strong to determine univocally the shape of the domain itself. The most famous overdetermined problem is of course the one solved by Serrin [21, where the overdetermination is written as $u=0$ and $|\nabla u|=$ constant on $\partial \Omega$, but also other kinds of overdetermined conditions have been considered in literature (see for instance $[1,2,5,6,9,13,22,23$ and the references therein). Here in Theorem 1.1 the overdetermination is given by the concavity property of the solution $u$ of (1.2), while in Theorem 1.2 the overdetermination is given by the existence of two homotethic level sets.

As a final remark, it has to be noted that the proof of Theorem 1.1 actually yields a much stronger result, namely Theorem 4.1 (see the end of Section 4). The latter may look less attractive than Theorem 1.1 but in fact it is precisely what we prove and Theorem 1.1 could be seen as a straightforward corollary of Theorem 4.1 ,

The paper is organized as follows. First, in Section 2, we recall some needed results and formulas (in particular the three main ingredients noted above). Section 3 contains the proof of Theorem 1.2, while Theorem 1.1 is proved in Section 4. Finally Section 5 is devoted to some final comments and remarks; in particular, it is shown that there exist convex sets different from a ball whose $p$-capacitary concavity number is greater than $-\infty$ and at the same time it is proved that there actually exist convex sets whose $p$-capacitary concavity number is precisely $-\infty$.

\section{Preliminaries}

2.1. Ingredient 1: the Brunn-Minkowski inequality for $p$-capacity. The original form of the Brunn-Minkowski inequality involves volumes of convex body (i.e. compact convex subsets of $\mathbf{R}^{n}$ with non-empty interior) and states that $\operatorname{Vol}_{n}(\cdot)^{1 / n}$ is a concave function with respect to the Minkowski addition, i.e.

$$
\left[\operatorname{Vol}_{n}\left(\lambda K_{1}+(1-\lambda) K_{2}\right)\right]^{\frac{1}{n}} \geq \lambda\left[\operatorname{Vol}_{n}\left(K_{1}\right)\right]^{\frac{1}{n}}+(1-\lambda)\left[\operatorname{Vol}_{n}\left(K_{2}\right)\right]^{\frac{1}{n}}
$$


for every convex body $K_{1}$ and $K_{2}$ and $\lambda \in[0,1]$. Here $\operatorname{Vol}_{n}$ is the $n$-dimensional Lebesgue measure and the Minkowski addition of convex sets is defined as follows:

$$
A+B=\{x+y \mid x \in A, y \in B\},
$$

while $\lambda A=\{\lambda x: x \in A\}$ for any $\lambda \in \mathbb{R}$, as usual.

Inequality (2.1) is one of the fundamental results in the modern theory of convex bodies; it can be extended to measurable sets and several other important inequalities, e.g. the isoperimetric inequality can be deduced from it.

Suitable versions of the Brunn-Minkowski inequality hold also for the other quermassintegrals (see [12,20]), and recently Brunn-Minkowski type inequalities have been proved for several important geometric and analytic functionals (see for instance the beautiful survey paper 12 by R. Gardner and possibly 19] for more recent references). Notice that in all the known cases, equality conditions are the same as in the classical Brunn-Minkowski inequality for the volume, i.e. equality holds if and only if the involved sets are (convex and) homothetic (i.e. translate and dilate of each other).

We will use the following theorem from [8].

Theorem 2.1 (Theorem 1, [8]). Let $K_{1}$ and $K_{2}$ be $n$-dimensional convex bodies and let $p \in(1, n)$. Then

$$
\left[\operatorname{Cap}_{p}\left(\lambda K_{1}+(1-\lambda) K_{2}\right)\right]^{\frac{1}{n-p}} \geq \lambda\left[\operatorname{Cap}_{p}\left(K_{1}\right)\right]^{\frac{1}{n-p}}+(1-\lambda)\left[\operatorname{Cap}_{p}\left(K_{2}\right)\right]^{\frac{1}{n-p}},
$$

for every $\lambda \in[0,1]$. Moreover equality holds if and only if $K_{1}$ and $K_{2}$ are homothetic.

Roughly speaking (2.2) says that $\operatorname{Cap}_{p}(\cdot)^{\frac{1}{n-p}}$ is a concave function in the class of convex bodies endowed with the Minkowski addition. But what is most relevant to the present paper is the equality condition: equality holds in (2.2) if and only if $K_{1}$ and $K_{2}$ are homothetic.

We recall that in the case of the Newton capacity, i.e. for $p=2$ and $n \geq 3$, inequality (2.2) was proved by C. Borell [3] and more recently in [4] L.A. Caffarelli, D. Jerison and E.H. Lieb treated the equality case. In 8 the treatments of the inequality and of its equality case are unified and the results are extended to a generic $p \in(1, n)$.

2.2. Ingredient 2: an expression of $p$-capacity through the behavior at infinity of the potential. In the case $p=2(n \geq 3)$ it is well known that the following relation between the Newton capacity of a convex domain and the behavior at infinity of the newtonian potential holds:

$$
\operatorname{Cap}_{2}(\Omega)=(n-2) \omega_{n} \lim _{|x| \rightarrow \infty} u(x)|x|^{n-2},
$$

where $\omega_{n}$ denotes the surface area of the unit sphere in $\mathbb{R}^{n}$. An analogous relation holds in the generic case $p \in(1, n)$

$$
\operatorname{Cap}_{p}(\Omega)=c_{n, p}\left[\lim _{|x| \rightarrow \infty} u(x)|x|^{(n-p) /(p-1)}\right]^{p-1},
$$

where

$$
c_{n, p}=\left(\frac{n-p}{p-1}\right)^{p-1} \omega_{n}
$$

refer to [8] for instance. 
2.3. Ingredient 3: the $p$-capacity of a level set of the potential. Let $u$ be the $p$-capacitary potential of a domain $\Omega$ and set

$$
\Omega(t)=\left\{x \in \mathbb{R}^{n}: u(x) \geq t\right\}
$$

for $t \in(0,1]$. Then it is easily seen that the following holds:

$$
\operatorname{Cap}_{p}(\Omega(t))=t^{1-p} \operatorname{Cap}_{p}(\Omega) .
$$

Indeed, the $p$-capacitary potential $u_{t}$ of $\Omega(t)$ is given by $u_{t}(x)=t^{-1} u(x)$, as it can be trivially verified, and (2.5) follows directly from (2.4). Notice however that (2.5) holds in more generality than (2.4), since it follows also from

$$
\operatorname{Cap}_{p}(\Omega)=\int_{\partial \Omega}|\nabla u|^{p-1} d \sigma=\int_{\partial \Omega(t)}|\nabla u|^{p-1} d \sigma, \quad \text { for every } t \leq 1,
$$

which can be obtained by integration by parts and does not need convexity of level sets, which is instead useful to prove (2.4).

\section{Proof of Theorem 1.2}

Let $0<r<s \leq 1, \rho>1, \xi \in \mathbb{R}^{n}$ such that

$$
\Omega(r)=\rho \Omega(s)+\xi,
$$

that is, $\Omega(r)$ and $\Omega(s)$ are the homothetic superlevel sets of the statement.

Notice that, since $r<s$, it holds that

$$
\Omega(s) \subset \Omega(r) .
$$

For $t \in(0,1]$, let us denote by $u_{t}$ the $p$-capacitary potential of $\Omega(t)$, i.e. the solution of

$$
\left\{\begin{array}{l}
\operatorname{div}\left(\left|\nabla u_{t}\right|^{p-2} \nabla u_{t}\right)=0 \quad \text { in } \quad \mathbf{R}^{n} \backslash \Omega(t), \\
u_{t}(x)=1 \quad \text { in } \bar{\Omega}(t), \\
\lim _{|x| \rightarrow+\infty} u_{t}(x)=0 .
\end{array}\right.
$$

Then

$$
u_{t}(x)=\frac{u(x)}{t}, \quad x \in \mathbf{R}^{n} \backslash \Omega(t)
$$

In particular

$$
u_{r}(x)=\frac{u(x)}{r} \quad \text { for } x \in \mathbf{R}^{n} \backslash \Omega(r) \quad \text { and } \quad u_{s}(x)=\frac{u(x)}{s} \quad \text { for } x \in \mathbf{R}^{n} \backslash \Omega(s) .
$$

On the other hand by (3.1) it holds that

$$
u_{r}(x)=u_{s}\left(\frac{x-\xi}{\rho}\right),
$$

and we finally get

$$
u(x)=\frac{r}{s} u\left(\frac{x-\xi}{\rho}\right), \quad x \in \mathbf{R}^{n} \backslash \Omega(r) .
$$


Then by (2.4) the latter implies

$$
\begin{aligned}
\left(\frac{\operatorname{Cap}_{p}(\Omega)}{c_{n, p}}\right)^{\frac{1}{p-1}} & =\lim _{|x| \rightarrow \infty} u(x)|x|^{\frac{n-p}{p-1}}=\frac{r}{s} \lim _{|x| \rightarrow \infty} u\left(\frac{x-\xi}{\rho}\right)|x|^{\frac{n-p}{p-1}} \\
& =\frac{r}{s} \rho^{\frac{n-p}{p-1}} \lim _{|x| \rightarrow \infty} u\left(\frac{x-\xi}{\rho}\right)\left(\frac{|x-\xi|}{\rho}\right)^{\frac{n-p}{p-1}}\left(\frac{|x|}{|x-\xi|}\right)^{\frac{n-p}{p-1}} \\
& =\frac{r}{s} \rho^{\frac{n-p}{p-1}}\left(\frac{\operatorname{Cap}_{p}(\Omega)}{c_{n, p}}\right)^{\frac{1}{p-1}},
\end{aligned}
$$

whence

$$
\frac{r}{s}=\rho^{\frac{p-n}{p-1}}
$$

Moreover (3.2) entails

$$
\Omega(t)=\rho \Omega\left(\frac{s}{r} t\right)+\xi \text { for } t<r .
$$

Hence, by setting

$$
s_{0}=s, \quad s_{1}=r, \quad s_{k}=\left(\frac{r}{s}\right)^{k} s=\rho^{\frac{k(p-n)}{p-1}} s, \quad k=2,3, \ldots,
$$

it holds that

$$
\lim _{k \rightarrow \infty} s_{k}=0
$$

and

$$
\begin{aligned}
\Omega\left(s_{k}\right) & =\rho \Omega\left(s_{k-1}\right)+\xi=\rho^{2} \Omega\left(s_{k-2}\right)+\rho \xi+\xi=\ldots \\
& =\rho^{k} \Omega\left(s_{0}\right)+\xi \sum_{i=0}^{k-1} \rho^{i}=\rho^{k} \Omega(s)+\xi \frac{\rho^{k}-1}{\rho-1} .
\end{aligned}
$$

Now let $x, y \in \partial \Omega(s)$, i.e.

$$
u(x)=u(y)=s,
$$

and set

$$
\begin{aligned}
& x_{k}=\rho^{k} x+\xi \frac{\rho^{k}-1}{\rho-1}, \\
& y_{k}=\rho^{k} y+\xi \frac{\rho^{k}-1}{\rho-1} .
\end{aligned}
$$

Then

$$
\lim _{k \rightarrow \infty}\left|x_{k}\right|=\lim _{k \rightarrow \infty}\left|y_{k}\right|=\infty
$$

and (2.4) yields

$$
\lim _{k \rightarrow \infty} u\left(x_{k}\right)\left|x_{k}\right|^{\frac{p-1}{n-p}}=\left(\frac{\operatorname{Cap}_{p}(\Omega)}{c_{n, p}}\right)^{1 /(p-1)}=\lim _{k \rightarrow \infty} u\left(y_{k}\right)\left|y_{k}\right|^{\frac{p-1}{n-p}} .
$$

On the other hand

$$
u\left(x_{k}\right)=u\left(y_{k}\right)=s_{k}
$$

hence (3.5) reads

$$
\lim _{k \rightarrow \infty} s_{k}\left|x_{k}\right|^{\frac{p-1}{n-p}}=\lim _{k \rightarrow \infty} s_{k}\left|y_{k}\right|^{\frac{p-1}{n-p}}
$$


that is,

$$
\lim _{k \rightarrow \infty}\left(\rho^{\frac{k(p-n)}{p-1}} s\left|\rho^{k} x+\xi \frac{\rho^{k}-1}{\rho-1}\right|^{\frac{p-1}{n-p}}\right)=\lim _{k \rightarrow \infty}\left(\rho^{\frac{k(p-n)}{p-1}} s\left|\rho^{k} y+\xi \frac{\rho^{k}-1}{\rho-1}\right|^{\frac{p-1}{n-p}}\right),
$$

which implies

$$
\lim _{k \rightarrow \infty}\left|x+\xi \frac{1-\rho^{-k}}{\rho-1}\right|^{\frac{p-1}{n-p}}=\lim _{k \rightarrow \infty}\left|y+\xi \frac{1-\rho^{-k}}{\rho-1}\right|^{\frac{p-1}{n-p}} .
$$

Since $\rho>1$, the latter finally implies

$$
\left|x+\xi \frac{1}{\rho-1}\right|=\left|y+\xi \frac{1}{\rho-1}\right|=R,
$$

which means that $\Omega(s)$ is a ball or radius $R$ centered at the point $\xi /(1-\rho)$, i.e.

$$
\Omega(s)=B\left(\frac{\xi}{1-\rho}, R\right),
$$

and from (3.4) we easily obtain

$$
\Omega\left(s_{k}\right)=B\left(\frac{\xi}{1-\rho}, R \rho^{k}\right) \quad \text { for } k=1,2, \ldots
$$

Then $u$ is radial in $\mathbb{R}^{n} \backslash \overline{\Omega(s)}$ and, by analytic continuation, it is radial in $\mathbb{R}^{n} \backslash \bar{\Omega}$ and $\Omega$ is a ball.

\section{Proof of Theorem 1.1}

Let $u \in C\left(\mathbb{R}^{n}\right) \cap C^{2}\left(\mathbb{R}^{n} \backslash \bar{\Omega}\right)$ be the solution of (1.2) and

$$
q=(1-p) /(n-p) .
$$

First we notice that, thanks to the monotonicity of the $\alpha$-concavity property, if $u$ is $\alpha$-concave for some $\alpha \geq q$, then it is $q$-concave. Next we will proceed by proving that, if $u$ is $q$-concave, then all its level sets are homothetic (and finally the proof will be concluded thanks to Theorem 1.2 ).

Assume that

$$
v=u^{q} \quad \text { is convex in } \mathbb{R}^{n} .
$$

Hence for every $v_{0}, v_{1} \in \mathbb{R}$ and for every $\mu \in(0,1)$ it holds that

$$
\begin{aligned}
\left\{x \in \mathbb{R}^{n}: v(x) \leq(1-\mu) v_{0}+\mu v_{1}\right\} \supseteq & (1-\mu)\left\{x \in \mathbb{R}^{n}: v(x) \leq v_{0}\right\} \\
& +\mu\left\{x \in \mathbb{R}^{n}: v(x) \leq v_{1}\right\} .
\end{aligned}
$$

Now take $r, s \in(0,1]$, fix $\lambda \in(0,1)$ and set

$$
t=\left[(1-\lambda) r^{q}+\lambda s^{q}\right]^{1 / q} .
$$

By setting $v_{0}=r^{q}$ and $v_{1}=s^{q}$, we have

$$
t^{q}=(1-\lambda) v_{0}+\lambda v_{1}
$$

and

Then (4.1) reads

$$
\begin{aligned}
& \Omega(r)=\left\{v \leq r^{q}\right\}, \\
& \Omega(s)=\left\{v \leq s^{q}\right\}, \\
& \Omega(t)=\left\{v \leq t^{q}\right\} .
\end{aligned}
$$

$$
\Omega(t) \supseteq(1-\lambda) \Omega(r)+\lambda \Omega(s) .
$$


Thanks to the monotonicity of $p$-capacity with respect to set inclusion, the latter implies

$$
\operatorname{Cap}_{p}(\Omega(t)) \geq \operatorname{Cap}_{p}((1-\lambda) \Omega(r)+\lambda \Omega(s))
$$

and by chaining with the Brunn-Minkowski inequality for $p$-capacity (see Theorem 2.1) we obtain

$$
\operatorname{Cap}_{p}(\Omega(t))^{1 /(n-p)} \geq(1-\lambda) \operatorname{Cap}_{p}(\Omega(r))^{1 /(n-p)}+\lambda \operatorname{Cap}_{p}(\Omega(s))^{1 /(n-p)} .
$$

On the other hand, by (2.5) we have

$$
\begin{gathered}
\operatorname{Cap}_{p}(\Omega(r))=r^{1-p} \operatorname{Cap}_{p}(\Omega), \\
\operatorname{Cap}_{p}(\Omega(s))=s^{1-p} \operatorname{Cap}_{p}(\Omega)
\end{gathered}
$$

and

$$
\operatorname{Cap}_{p}(\Omega(t))=t^{1-p} \operatorname{Cap}_{p}(\Omega) .
$$

Substituting in (4.3) and taking into account (4.2), we finally get

$$
\operatorname{Cap}_{p}(\Omega(t))^{1 /(n-p)}=(1-\lambda) \operatorname{Cap}_{p}(\Omega(r))^{1 /(n-p)}+\lambda \operatorname{Cap}_{p}(\Omega(s))^{1 /(n-p)},
$$

i.e. equality holds in (4.3), and consequently equality must hold in the BrunnMinkowski inequality for $p$-capacity for $\Omega(r)$ and $\Omega(s)$. Then Theorem 2.1 tells that $\Omega(r)$ and $\Omega(s)$ must be homothetic. This concludes the proof thanks to Theorem 1.2, as already said.

As a final remark of this section, we notice that in fact in the previous proof, we did not use the full strength of $q$-concavity of $u$; that is, we do not use (4.1) for every combination of $\mu, v_{0}$ and $v_{1}$, but it is actually sufficient to have it for a fixed triplet $\lambda, r, s$ in order to obtain the full result. Then we actually proved the following strengthened version of Theorem 1.1 .

Theorem 4.1. Let $p \in(1, n), q=(1-p) /(n-p), \Omega$ be a bounded domain in $\mathbb{R}^{n}$ (not necessarily convex) and $u$ be the solution of (1.2). If $u$ has three convex super level sets

$$
\begin{gathered}
\Omega(r)=\left\{x \in \mathbb{R}^{n}: u(x) \geq r\right\}, \Omega(s)=\left\{x \in \mathbb{R}^{n}: u(x) \geq s\right\}, \\
\Omega(t)=\left\{x \in \mathbb{R}^{n}: u(x) \geq t\right\}
\end{gathered}
$$

$($ say $0<s<r \leq 1)$ such that

$$
t \geq\left[(1-\lambda) r^{q}+\lambda s^{q}\right]^{1 / q}
$$

and

$$
\Omega(t) \supseteq(1-\lambda) \Omega(r)+\lambda \Omega(s)
$$

for some $\lambda \in(0,1)$, then $\Omega$ is a ball.

The proof is precisely the same as above, as already said. Let us just point out that here we do not need to assume the convexity of $\Omega$; however, we have to assume the convexity of $\Omega(r)$, which yields the convexity of $\Omega(s)$ and $\Omega(t)$ (in fact of $\Omega(\tau)$ for every $\tau \leq r$, but not of $\Omega$ itself), in order to apply Theorem 2.1. That has been proved only for convex sets up to now. 


\section{Final COMments and REmarks}

So far we know that for every convex set $\Omega$ the associated $p$-potential $u$ is quasiconcave, which allows us to define the $p$-concavity number $\alpha(\Omega, p)$ and in the case of a ball we can explicitly calculate $\alpha(B, p)=(1-p) /(n-p)$. Then we have proved here that this value is the maximum possible and that it is realized only by the balls. On the other hand, one could wonder whether the ball is the only one set such that $\alpha(\Omega, p)>-\infty$, or even, on the contrary, if it happens that $\alpha(\Omega, p)>-\infty$ for every convex set. The answer to both these questions is negative.

Proposition 5.1. Let $n=3$. Then there exist infinitely many convex sets such that $\alpha(\Omega, 2) \geq-2$.

Proof. Let $n \geq 3, p>1, q=(n-p) /(p-1), B=\left\{x \in \mathbb{R}^{n}:|x|<1\right\}$ be the unit ball and $u(x)=|x|^{-q}$ its $p$-potential. Then we notice that $v(x)=u(x)^{-2 / q}=|x|^{2}$ is uniformly convex in $\mathbb{R}^{n} \backslash B$ since

$$
D^{2} v=2 I \text { for every } x \in \mathbb{R}^{n} \backslash B,
$$

where $I$ denotes the $n \times n$ identity matrix.

Now let $n=3$ and $p=2$ (whence $q=1$ ), take $a, b, c>0$ and denote by $\Omega(a, b, c)$ the ellipsoid with axes of length $a, b$ and $c$, i.e.

$$
\Omega(a, b, c)=\left\{(x, y, z) \in \mathbb{R}^{3}: \frac{x^{2}}{a^{2}}+\frac{y^{2}}{b^{2}}+\frac{z^{2}}{c^{2}}<1\right\} .
$$

Then the Newton potential $u_{a b c}$ of $\Omega(a, b, c)$ can be computed as in 15, Section 6 - formula (33)] and it can be seen to depend smoothly (together with all its derivatives) on $a, b$ and $c$. Then by choosing any $\epsilon \in(0,1)$, by (5.1) for $a, b, c$ close enough to 1 we have $D^{2}\left(u_{a b c}^{-2}\right) \geq \epsilon I$, which means that $u_{a b c}$ is $(-2)$-concave, i.e. $\alpha(\Omega(a, b, c), 2) \geq-2$.

Actually, even for generic $n \geq 3$ and $p>1$, thanks to (5.1) if a smooth convex domain $\Omega$ is close to a ball in some strong sense so that its potential $u$ is $C^{2}$-close enough to the potential of the ball, then $\alpha(\Omega, p) \geq-2 q$.

Proposition 5.2. Let $n \geq 3$ and $p>1$. There exist infinitely many convex sets such that the associated p-capacitary potential is genuinely quasi-concave and nothing more, that is, $\alpha(\Omega, p)=-\infty$.

Proof. Consider a hypercube $Q=\left\{x=\left(x_{1}, \ldots, x_{n}\right):\left|x_{i}\right|<1 i=1, \ldots, n\right\}$ and let $u$ be its $p$-capacitary potential. Then it is easily seen by a barrier argument (see for instance [18, Section 4.2]) that $\nabla u$ blows up on the vertices and edges of $Q$ (in fact at every singular point of $\partial Q$ ). This prevents $u$ from being $\alpha$-concave for any $\alpha>-\infty$, since the gradient of $u^{\alpha}$ has to blow up at the same points (and this contradicts the monotonicity property of the gradient of a convex function).

The argument obviously works for every convex polytope.

According to the above argument, the main obstacle to power concavity of the potential seems to be the lack of regularity of $\partial \Omega$. Then let us state the following conjecture, after recalling that a convex domain is said to be of class $C_{+}^{2}$ if it has a $C^{2}$ boundary whose Gauss curvature never vanishes.

Conjecture. $\alpha(\Omega, p)>-\infty$ for every $\Omega$ of class $C_{+}^{2}$ (and for every $p>1$ ). 
In conclusion, a final natural question of whether for every $\alpha \in[-\infty,-1 / q]$ (and for every $p>1$ ) there exist convex sets whose $p$-capacitary concavity number is precisely $\alpha$ remains open. To investigate this question in more detail is beyond the scope of this short note, but we would guess the answer is affirmative (and Propositions 5.1 and 5.2 give some hints in this direction).

\section{REFERENCES}

[1] Virginia Agostiniani and Rolando Magnanini, Symmetries in an overdetermined problem for the Green's function, Discrete Contin. Dyn. Syst. Ser. S 4 (2011), no. 4, 791-800, DOI 10.3934/dcdss.2011.4.791. MR2746441(2012a:35211)

[2] Chiara Bianchini, A Bernoulli problem with non-constant gradient boundary constraint, Appl. Anal. 91 (2012), no. 3, 517-527, DOI 10.1080/00036811.2010.549479. MR2876741

[3] Christer Borell, Capacitary inequalities of the Brunn-Minkowski type, Math. Ann. 263 (1983), no. 2, 179-184, DOI 10.1007/BF01456879. MR698001 (84e:31005)

[4] Luis A. Caffarelli, David Jerison, and Elliott H. Lieb, On the case of equality in the Brunn-Minkowski inequality for capacity, Adv. Math. 117 (1996), no. 2, 193-207, DOI 10.1006/aima.1996.0008. MR.1371649 (97f:31011)

[5] Andrea Cianchi and Paolo Salani, Overdetermined anisotropic elliptic problems, Math. Ann. 345 (2009), no. 4, 859-881, DOI 10.1007/s00208-009-0386-9. MR2545870(2010i:35264)

[6] G. Ciraolo, R. Magnanini, S. Sakaguchi, Symmetry of minimizers with a level surface parallel to the boundary, preprint, 2012. arXiv:1203.5295v2

[7] Andrea Colesanti, Brunn-Minkowski inequalities for variational functionals and related problems, Adv. Math. 194 (2005), no. 1, 105-140, DOI 10.1016/j.aim.2004.06.002. MR2141856 (2006a:35047)

[8] Andrea Colesanti and Paolo Salani, The Brunn-Minkowski inequality for p-capacity of convex bodies, Math. Ann. 327 (2003), no. 3, 459-479, DOI 10.1007/s00208-003-0460-7. MR2021025 (2004j:31007)

[9] Cristian Enache and Shigeru Sakaguchi, Some fully nonlinear elliptic boundary value problems with ellipsoidal free boundaries, Math. Nachr. 284 (2011), no. 14-15, 1872-1879, DOI 10.1002/mana.200810170. MR2838287(2012k:35369)

[10] Lawrence C. Evans and Ronald F. Gariepy, Measure theory and fine properties of functions, Studies in Advanced Mathematics, CRC Press, Boca Raton, FL, 1992. MR.1158660 (93f:28001)

[11] R. M. Gabriel, A result concerning convex level surfaces of 3-dimensional harmonic functions, J. London Math. Soc. 32 (1957), 286-294. MR0090662 (19,848a)

[12] R. J. Gardner, The Brunn-Minkowski inequality, Bull. Amer. Math. Soc. (N.S.) 39 (2002), no. 3, 355-405, DOI 10.1090/S0273-0979-02-00941-2. MR1898210 (2003f:26035)

[13] Antoine Henrot and Gérard A. Philippin, Some overdetermined boundary value problems with elliptical free boundaries, SIAM J. Math. Anal. 29 (1998), no. 2, 309-320 (electronic), DOI 10.1137/S0036141096307217. MR1616562 (99b:35159)

[14] Bernhard Kawohl, Rearrangements and convexity of level sets in PDE, Lecture Notes in Mathematics, vol. 1150, Springer-Verlag, Berlin, 1985. MR810619 (87a:35001)

[15] Oliver Dimon Kellogg, Foundations of potential theory, Reprint from the first edition of 1929. Die Grundlehren der Mathematischen Wissenschaften, Band 31, Springer-Verlag, Berlin, 1967. MR0222317 (36 \#5369)

[16] Alan U. Kennington, Power concavity and boundary value problems, Indiana Univ. Math. J. 34 (1985), no. 3, 687-704, DOI 10.1512/iumj.1985.34.34036. MR794582 (87c:35056)

[17] John L. Lewis, Capacitary functions in convex rings, Arch. Rational Mech. Anal. 66 (1977), no. 3, 201-224. MR0477094 (57 \#16638)

[18] Osvaldo Mendez and Wolfgang Reichel, Electrostatic characterization of spheres, Forum Math. 12 (2000), no. 2, 223-245, DOI 10.1515/form.2000.005. MR.1740890 (2001a:31005)

[19] Paolo Salani, Convexity of solutions and Brunn-Minkowski inequalities for Hessian equations in $\mathbb{R}^{3}$, Adv. Math. 229 (2012), no. 3, 1924-1948, DOI 10.1016/j.aim.2011.12.009. MR2871162

[20] Rolf Schneider, Convex bodies: the Brunn-Minkowski theory, Encyclopedia of Mathematics and its Applications, vol. 44, Cambridge University Press, Cambridge, 1993. MR.1216521 (94d:52007) 
[21] James Serrin, A symmetry problem in potential theory, Arch. Rational Mech. Anal. 43 (1971), 304-318. MR0333220 (48 \#11545)

[22] Philip W. Schaefer, On nonstandard overdetermined boundary value problems, Proceedings of the Third World Congress of Nonlinear Analysts, Part 4 (Catania, 2000), Nonlinear Anal. 47 (2001), no. 4, 2203-2212, DOI 10.1016/S0362-546X(01)00345-5. MR1971630 (2004b:35076)

[23] H. Shahgholian, Diversifications of Serrin's and related symmetry problems, Complex Var. Elliptic Equ. 57 (2012), no. 6, 653-665. MR2916825

Dimai - Departimento di Matematica e Informatica "U. Dini", Università degli Studi di Firenze, Viale Morgagni 67/A, 50134 Firenze, Italy

E-mail address: paolo.salani@unifi.it 